\section{Reactions in theory}

\author{
P.E. Hodgson
}

Direct Nuclear Reactions.

By G.R. Satchler.

Oxford University Press: 1983. Pp.833.

$£ 55, \$ 110$.

Direct Nuclear Reactions.

By Norman K. Glendenning.

Academic: 1983. Pp.378. \$62, £45.50.

FOR the past 20 years the names of Ray Satchler and Norman Glendenning have been familiar to all students of nuclear reactions. These two men pioneered the development of practically every aspect of the theoretical formalism widely used to analyse experimental data and to extract information on nuclear structure. Both, inevitably, have been invited to write books. Ray Satchler first agreed to write his in 1960; Norman Glendenning began thinking about it in 1969 but did not start writing until 1978. And now the two books are published together.

Direct nuclear reactions are those processes that take place in the initial stages of a nuclear collision, in the time it takes the projectile to traverse the field of the target nucleus. Rather few degrees of freedom are excited by such processes, so that studies of the energies and angular distributions of the outgoing particles yield important information on nuclear structure. Subsequently, other processes take place, often with further particle emission.

Broadly speaking, there are three types of direct reactions - elastic scattering that leaves the target nucleus unchanged; inelastic scattering that raises it to an excited state; and transfer reactions that produce a different nucleus. Inelastic scattering tends to excite collective states in which many nucleons move coherently, while transfer reactions preferentially excite states that can be described as the excitation of one nucleon or group of nucleons. Thus different types of reactions can be used to explore very different types of nuclear excitation.

The physical ideas underlying our current understanding of these reaction processes are mostly quite simple, but their quantum mechanical formulation is frequently a task of formidable complexity and the resulting equations can only be solved by powerful computers. Both authors are theoreticians, with complete mastery of the formal techniques of reaction analysis and the angular momentum algebra needed in realistic calculations. So it is no surprise that the main part of each book is devoted to accounts of the formalisms appropriate to different types of direct reactions, and the approximations necessary to obtain reasonably accurate results in finite computing time.

There is inevitably much common ground between the two books, but with differences of emphasis and depth of treat- ment. Both cover the basic reaction formalism, starting with the general expression for the transition amplitude, partial wave analysis and the optical model. Next in order of complexity comes the distorted wave theory of one-nucleon transfer reactions. Both books cover this in considerable detail, going on to describe the coupled-channel formalism that makes it possible to include higher order processes that are important when the coupling between channels is strong. This is essential to the understanding of the excitation of collective states by many different projectiles. The same technique may be applied to twonucleon and multi-nucleon transfer reactions, with considerable increase in formal complexity. It is also possible to develop microscopic theories of the reactions, in which the participating nucleons are considered separately. Finally, the collisions of nuclei with each other, known as heavy-ion interactions, reveal a wealth of new phenomena that may be understood by extending the concepts and formalism developed for the interactions of the lighter particles.

On the whole, both books start from a level appropriate to the graduate student. Glendenning keeps to essentials, while Satchler describes many more detailed applications and special techniques. Both authors give many references to the original literature, and illustrate their discussions by comparisons with experimental data.

The scope of both books is almost entirely confined to direct reactions, with little mention of the subsequent pre-equilibrium and compound nucleus processes, or of their relative importance at different energies and for different projectiles and

\section{Diffusing knowledge}

\section{Charles DeLisi}

Random Walks in Biology.

By Howard C. Berg.

Princeton University Press: 1984. Pp.142. $\$ 16.50$, f15.30.

A GOOD swimmer can typically coast about $18 \mathrm{ft}$, approximately three body lengths. A bacterium, on the other hand, once it stops propelling itself, will come to rest in $0.4 \AA$ - almost two orders of magnitude less than the diameter of a hydrogen atom. The inertial equivalent of a bacterium moving through water, is Homo sapiens swimming in butter! Evidently, an intuition about physical processes that occur on scales characteristic of our daily environment does not always extrapolate readily to the world of the very small. The purpose of Howard Berg's book is to sharpen our intuition about the physics and statistics that govern motion in the microscopic environment of the cell.

The random molecular motions of the microscopic world that manifest them- target nuclei. The experimentalist is however confronted with a cross-section, and the first question he has to answer is which of these processes has occurred. Only when he knows the answer, at least provisionally, can he begin to analyse the data using the appropriate theory. The usefulness of the books would have been somewhat increased by more consideration of this problem.

A more subtle question encountered in the analysis of data is the degree of flexibility of the theories used. It is often relatively easy, by judicious parameter adjustment, to fit a theory to a known crosssection, but it is much harder to predict with confidence the value of a cross-section before it has been measured. It is notoriously difficult to judge the flexibility of a theory just by reading other people's papers - one has to repeat the analysis for oneself. Both authors are familiar with this problem, and it would have been valuable to learn more about their own experiences. One final - and minor - comment; selectivity is inevitable in works such as these, but in both books more references to peripheral areas of research would have been welcome, especially to proceedings of conferences and summer schools.

Satchler and Glendenning have undoubtedly earned the gratitude of nuclear physicists, and their books will be essential for reference and study. These volumes should be in every science library, and research workers would do well to save up until they can afford their own copies. $\square$

P.E. Hodgson is Head of the Nuclear Physics Theoretical Group at the Nuclear Physics Laboratory, University of Oxford.

selves by macroscopic regularity dominate both the motion of cells and the techniques used to study them. It is well known that the regular movement of a sedimentation band finds its microscopic explanation in the random movement of individual molecules. The effect of Brownian motion on the ability of a cell to capture efficiently ligands in its environment is, however, less well known. Who would guess that with only a fraction of a per cent of a cell surface covered by receptors, the rate at which ligands are captured can be nearly half that of a uniformly absorbing sphere!

Random Walks in Biology, which supplements and elaborates on the now classic paper by Berg and Edward Purcell ("Physics of Chemoreception" - Biophys. J. 20, 193-219; 1977), is a scholarly and pedagogically masterly introduction to diffusion, its physics and its statistics. The book will probably require several readings in order fully to assimilate its concepts, but the time will be well and enjoyably spent.

Charles DeLisi is Chief of the Laboratory of Mathematical Biology, National Institutes of Health, Bethesda, Maryland. 